\title{
DETERMINATION OF POISSON'S RATIO BY MEANS OF AN INTERFERENCE APPARATUS.
}

By J. R. Benton.

POISSON proved that when a homogeneous isotropic body is extended in one direction, it must contract in the directions perpendicular to the extension, and that if the intermolecular forces are assumed equal in all directions, the contraction must amount to $1 / 4$ of the extension. Numerous experiments, embracing a great variety of methods, have shown that this contraction actually does take place, but that the ratio in which it stands to the extension-Poisson's ratio-is usually different from $1 / 4$. This is accounted for under the assumption of imperfect homogeneity; indeed the ratio varies greatly for the same material according to the manner in which it has been treated. Voigt, ${ }^{1}$ experimenting on electrolytically deposited copper, where there is no reason to expect inhomogeneity, found the ratio to have almost exactly its theoretical value of $1 / 4$.

The methods of determining Poisson's ratio may be divided into two classes.

First, those which depend on a comparison of the elastic constants of the medium investigated. It can be shown that Poisson's ratio $K$ satisfies the equation :

$$
K=\frac{B}{A+2 B}
$$

where $A$ and $B$ are fundamental elastic constants of the medium. $A$ and $B$ can be determined from the behavior of the medium with respect to two different kinds of deformations, such as extension and flexure. Thus extension and torsion were used by Kohlrausch and Loomis, ${ }^{2}$ and by Baumeister ${ }^{3}$ to determine $K$; flexure and torsion

${ }^{1}$ W. Voigt: Berliner Sitzungsberichte, 1884, p. 961 ; 1884, p. 1004 .

2 Poggendorft's Annalen, Band 14r, p. 502, 1870.

'Wiedemann's Annalen, Band 18, p. 578, 1883 . 
by Voigt, ${ }^{1}$ Kirchhoff ${ }^{2}$ and Okatow, ${ }^{3}$ while Amagat ${ }^{4}$ compared with other deformations cubical contraction under increase of pressure. Schneebelli ${ }^{5}$ compared the velocity of torsional and longitudinal vibration of metal rods. Cornu ${ }^{6}$ used an ingenious method in investigating Poisson's ratio for glass; one piece of glass was slightly bent by means of weights, and another piece was laid on it ; the resulting interference bands had the form of hyperbolas, and it can be shown that the tangent of the angle between the asymptotes of the hyperbolas and the axis of the glass plate is equal to Poisson's ratio.

Second, Poisson's ratio may be determined by measuring the contraction at right angles to the direction of the extension, and comparing it with the extension. The former of these quantities is very small, and has to be measured indirectly, unless the stretched body is a very thick rod, in which case larger forces than are usually available have to be applied to produce an appreciable extension. The problem reduces itself, therefore, to that of the measurement of a very small distance.

The first determination, carried out by Cagniard de la Tour ${ }^{7}$ to test Poisson's theoretical result, was made by stretching a wire contained in a narrow tube filled with water. The contraction of the wire was calculated from the change of level of the water. A similar method was employed by Wertheim, ${ }^{8}$ who made important investigations of Poisson's ratio; he filled a hollow cylinder, communicating with a capillary tube, with water; when the cylinder was stretched, the change of its volume could be determined from the change of level of the water in the capillary tube, and from this Poisson's ratio was obtained. Both these methods were unsatisfactory, because the apparatus acted like delicate thermometers, a very slight change of temperature of the large mass of water making a great difference in its level in the narrow tube.

1 W. Voigt, Wiedemann's Amalen, Band 15, p. 497, 1882.

2 Pogg. Annalen, Band 108, p. 369, I859.

3 Pogg. Annalen, Band I19, p. II, 1863 .

4 Comp. Rend., 2I, July, 1884.

${ }^{5}$ Pogg. Annalen, Band 140, p. 589, 1870.

${ }^{6}$ A. Cornu, Comptes Rendus, Tome 69, p. 333, r869.

${ }^{7}$ Ann. Chim. Phys., Tome 36, p. $3^{84}, 1827$.

${ }^{8}$ Ann. Chim. Phys., 3 ser., Tome 23, p. 52. 
[Vol. XII.

Measurements by means of the microscope have been made by Mallock, ${ }^{1}$ who determined the change in distance between points marked on a rod, when the rod was bent. Direct measurement of the contraction has been made in cases where the deformation was very great ; thus Röntgen ${ }^{2}$ determined Poisson's ratio for caoutchouc by stamping a circle on a stretched band; when the tension was removed the circle became an ellipse whose axes furnished the necessary data for the determination.

At the suggestion of Professor Voigt, I undertook the measurement of the contraction by means of interference bands. The experiments were made in his laboratory in the University of Göttingen.

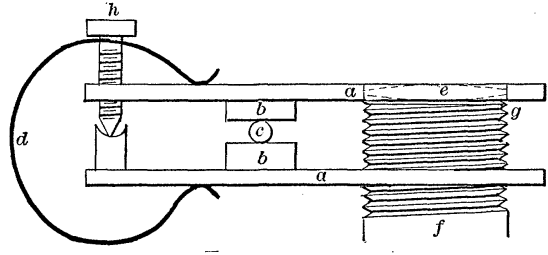

View from above.
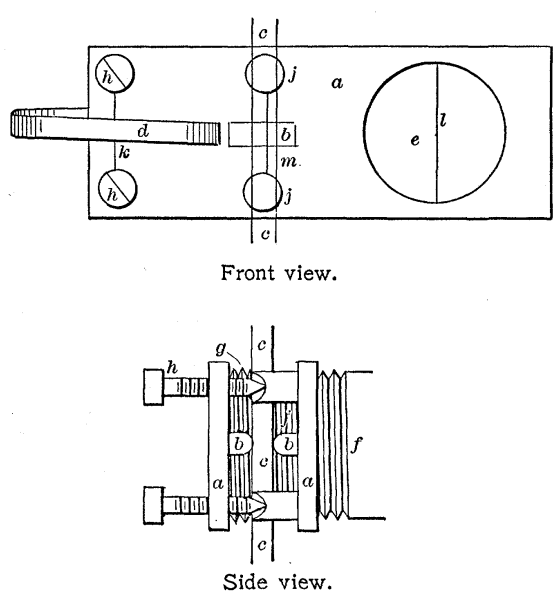

Fig. 1.

The interference of light has been already used to determine Poisson's ratio. Stromeyer and Kennedy ${ }^{3}$ made use of it to measure the contraction of thick metal bars which were stretched in a testing machine. My apparatus is designed for use where such large forces are not available.

After making attempts with numerous variations of the apparatus, the following was found to give the most satisfactory results. $a, a$ (Fig. I) were plates of brass, on which the rounded bits of steel $b$, $b$ were soldered, and firmly pressed against the wire $c$ by means of the spring $d$. One plate contained a lens $e$, of slight curvature, the other a tube $f$, having a screw thread so that the distance of the glass

1 Proc. Roy. Soc., vol. 29, p. 157, 1879.

2 Poggendorff's Annalen, Band r57, p. 601, 1876.

3 Proc. Roy. Soc., vol. 55, pp. 373-383, 1894. 
plate $g$ in its end from the lens could be adjusted. The back side of the glass plate $g$ was blackened. By means of the screws $h$, $h$ it was possible to set the plate perpendicular to the axis of the lens; in which case interference rings appeared, provided the distance between lens and plate was not too great. If the wire contracted, the distance became less, and new rings appeared. If sodium light is used, each new ring corresponds to a difference of distance of $294.5 \times 10^{-6} \mathrm{~mm}$., the half wave length of the light. This difference is not, however, itself the contraction of the wire, but the latter must be deduced from it when the distance of the wire and the center of the interference rings from the axis are known, around which the brass plates move. This axis is simply the line passing through the points of the screws $h, h$. In order to measure the distance from this axis, I ruled the fine lines $k, l$ and $m$ and measured accurately the distances $k l$ and $k m$, once for all. Then in any particular case the distances from $l$ to the center of the rings, and from $m$ to the center of the wire (seen through the holes $j j$ ) were measured by means of a microscope, and added to or subtracted from $\mathrm{kl}$ and $\mathrm{km}$.

The wire was fastened above a table $a$ (Fig. 2) and hung through a hole in it. $b$ was a strong piece of iron. Underneath, the hook $a^{\prime}$, carrying the scale pan $e$, hung from a loop in the wire. A stiff iron $\operatorname{rod} f$, free to move round the horizontal axis $g$, passed through a hole in the rod $h$ and carried a weight $l$. The purpose of the weights $e$ and $l$ was simply to hold the wire tight, and to prevent $f$ from slipping in the hole $h$. The weight by which the tension of the wire was produced, was applied to the end of $f$.

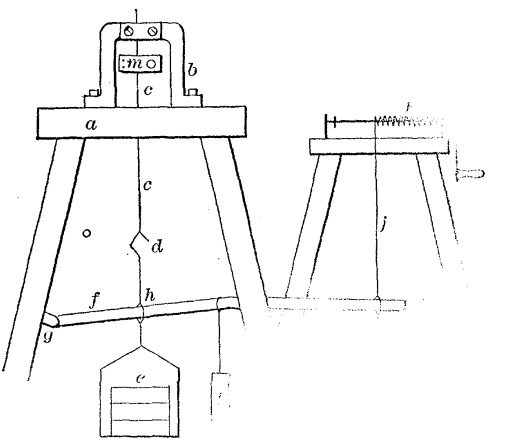

Fig. 2. The weight $i$ wasplaced on a scale pan, which could be lowered by the windlass $k$ so as to the rest on $f$, or raised from it ; the purpose of this arrangement being to avoid jars in changing the weights. The interference apparatus was at $m:$ in front of it was a Bunsen burner 
(not shown in the figure), in which a bead of $\mathrm{Na}_{2} \mathrm{CO}_{3}$ was held, and a microscope (also not shown), with which the rings were observed. The angle between the incident and the reflected ray was very small.

Each wire, after being fastened in the iron $b$, was heated to dull redness under a small load, by means of a Bunsen burner, in order to insure its hanging straight. Then it was left a day or two under a somewhat greater load than the one to be used in the experiments, after which the measurements were made.

The interference apparatus was placed on a wire, and the screws $h h$ (Fig. I), and the tube $f$ so adjusted that the plate $g$ was perpendicular to the axes of the lens, and very near to it, but yet not so near as to come into actual contact with it when the wire contracted. The cross-hairs of the microscope were set at first on the center of the rings which appeared under these conditions. Then the microscope was clamped in position, and the micrometer screw-head was turned until the intersection of the cross-hairs was on the tenth ring above the center. Here five readings of the micrometer were made, then the cross-hairs were brought on to the tenth ring underneath, where five readings were taken; then five readings were taken again on the tenth ring above. The difference between the average reading above and below gives the diameter of the tenth ring in terms of the divisions on the screw-head. Then the tension was brought to bear on the wire, the number of new rings counted, and the diameter of the tenth ring under these conditions was measured. Finally the tension was removed, another measurement of the diameter of the tenth ring was made, and also its difference from the diameter of the ninth ring and from that of the eleventh determined. The difference in diameter between the tenth ring with and without tension, divided by the difference in diameter between the tenth ring and the ring next to it, gives the fraction which must be added to the number of new rings in order to determine the change of distance between the plate and the lens. Suppose, for example, that the diameter (in terms of the divisions on the screw-head) of the tenth ring is $\mathrm{I} 8.22 \mathrm{I}$ with tension, $\mathrm{I} 8.005$ without tension; that the average of the difference in diameter between the ninth and tenth, and between the tenth and eleventh rings is 0.920 ; and that 4 new rings have appeared, then the distance through which the lens and plate have approached each other is 


$$
294.5 \times 10^{-6} \times\left(4+\frac{18.22 \mathrm{I}-\mathrm{I} 8.005}{0.920}\right)=\mathrm{I} 247 \times 10^{-6} \mathrm{~mm} .
$$

After making two such determinations at different places on the wire, it was loosened, turned through $90^{\circ}$, fastened again, heated to dull redness, and left hanging under a heavy load for a few hours. Then similar determinations were made at two different places on the wire, so that for each wire four determinations were made. The results given below are the averages of four such determinations each. The distance of the wire and rings from the screws $h h$ (Fig. I) was measured as described before; the weight $i$ (Fig. 2) and the scale pan were weighed in a balance; the distance of the wire and the weight from the axis $g$ were measured with a millimeter scale; and the diameter of the wire was measured with accurate calipers, the mean of six measurements being taken. From these quantities the contraction of the wire due to unit tension can be calculated.

Poisson's ratio is obtained by dividing this contraction by the extension due to unit tension. The last-named quantity was determined by measuring, with a micrometer, the vertical distance through which a needle point fastened to the end of the wire moved when weights were hung on the wire. For this purpose a 3-meter length of wire was used.

The values of Poisson's ratio found for a number of wires are given in the following table:

\begin{tabular}{l|c|c|c}
\hline \multicolumn{1}{c|}{ Substance. } & Diameter. & Poisson's Ratio. & Mean Value. \\
\cline { 2 - 3 } Steel. & $0.685 \mathrm{~mm}$. & 0.276 & \\
& 1.405 & 0.275 & 0.2755 \\
Iron. & 0.948 & 0.294 & \\
& 1.578 & 0.282 & 0.288 \\
Copper. & 1.390 & 0.337 & \\
Nickel. & 1.728 & 0.345 & 0.341 \\
& 0.963 & 0.375 & \\
Brass. & 1.490 & 0.271 & \\
Nickelin. & 0.787 & 0.328 & \\
& 1.498 & 0.334 & 0.331 \\
German Silver. & 0.978 & 0.406 & \\
Bronze. & 1.397 & 0.399 & 0.403 \\
Brass containing & 1.005 & 0.313 & \\
much copper. & 1.592 & 0.429 & \\
\hline
\end{tabular}


The highly unsatisfactory discordant results for nickel appear to be due to some difference in the physical condition of the two wires. I found that their specific gravities were somewhat different. Of course the experiments were repeated, as soon as the discrepancy was found, but the same results were obtained.

The difference between the two values for German silver is due to difference in the composition of the alloy for the two wires. 